\title{
Saccharification and ethanol fermentation from cholinium \\ ionic liquid-pretreated bagasse with a different number of \\ post-pretreatment washings
}

Kazuaki Ninomiya $^{\mathrm{a}}$, Sayuri Omote ${ }^{\mathrm{b}}$, Chiaki Ogino ${ }^{\mathrm{c}}$, Kosuke Kuroda ${ }^{\mathrm{b}}$, Mana Noguchi ${ }^{\mathrm{b}}$,

Takatsugu Endo ${ }^{\mathrm{b}}$, Ryohei Kakuchi ${ }^{\mathrm{b}}$, Nobuaki Shimizu ${ }^{\mathrm{a}}$, and Kenji Takahashi ${ }^{\mathrm{b}, *}$

${ }^{a}$ Institute of Nature and Environmental Technology, Kanazawa University,

Kakuma-machi, Kanazawa 920-1192, Japan

${ }^{b}$ Faculty of Natural System, Institute of Science and Engineering, Kanazawa University, Kakuma-machi, Kanazawa 920-1192, Japan

${ }^{c}$ Department of Chemical Science and Engineering, Graduate School of Engineering, Kobe University, 1-1 Rokkodaicho, Nada-ku, Kobe 657-8501, Japan

${ }^{*}$ Corresponding author: Kenji Takahashi

Faculty of Natural System, Institute of Science and Engineering, Kanazawa University, Kakuma-machi, Kanazawa 920-1192, Japan

Tel.: +81 76234 4828; Fax: +81 762344829

E-mail address: ktkenji@ staff.kanazawa-u.ac.jp 


\begin{abstract}
Choline acetate (ChOAc), a cholinium ionic liquid (IL), was compared with 1-ethyl-3-methylimidazolium acetate (EmimOAc) with regard to biomass pretreatment, inhibition on cellulase and yeast, residuals in pretreated biomass, and saccharification and fermentation of pretreated biomass. Irrespective of ChOAc and EmimOAc, cellulose and hemicellulose saccharification of the IL-pretreated bagasse were over $90 \%$ and $60 \%$, respectively. Median effective concentrations $\left(\mathrm{EC}_{50}\right)$ based on cellulase activity were $32 \mathrm{wt} \%$ and $16 \mathrm{wt} \%$ for ChOAc and EmimOAc, respectively. The $\mathrm{EC}_{50}$ based on yeast growth were $3.1 \mathrm{wt} \%$ and $0.3 \mathrm{wt} \%$ for ChOAc and EmimOAc respectively. The residuals in IL-pretreated bagasse were $10 \%$ and $23 \%$ for ChOAc and EmimOAc, respectively, when washed 2 times after pretreatment. Ethanol yield on a bagasse basis were $60 \%$ and $24 \%$ for ChOAc and EmimOAc, respectively, in the saccharification and fermentation of IL-pretreated bagasse when washed 2 times. ChOAc-pretreated bagasse could be saccharified and fermented with fewer wash times than EmimOAc-pretreated bagasse.
\end{abstract}

\title{
Keywords:
}

Cholinium, Ionic liquid, Pretreatment, Lignocellulose, Wash times 


\section{Introduction}

In this decade, ionic liquids (ILs; generally defined as organic salts that melt below $100^{\circ} \mathrm{C}$ ) have been the focus of considerable attention owing to their thermal stability, non-volatility, and capability of dissolving various polymeric compounds. It was previously demonstrated that some ILs can dissolve cellulose (Swatloski et al., 2002) and that the re-precipitated cellulose showed a higher efficiency of enzymatic saccharification because the crystallinity was significantly decreased (Dadi et al., 2006). This IL-assisted pretreatment has been applied toward several types of lignocellulosic materials (Brandt et al., 2013), since the IL-assisted pretreatment has been demonstrated to be more effective than the conventional pretreatment using diluted acid or ammonia (Li et al., 2010, 2011).

After the IL-assisted pretreatment step, the pretreated biomass needs to be washed extensively to remove the residual IL since the residual IL in pretreated biomass inhibits cellulolytic enzymes and fermentative microbes in the subsequent saccharification and fermentation step (Datta et al., 2010; Ouellet et al., 2011). This extensive washing of pretreated biomass results in large amounts of diluted IL aqueous solution, which leads to high costs for both concentrating IL from its diluted aqueous solution by evaporation and treating the resultant wastewater.

Recently, completely bio-derived cholinium ILs were reported, which contain either cholinium cations combined with amino acid-based anions (Hu et al., 2007) or carboxylic acid-based anions (Fukaya et al., 2007). Moreover, it was demonstrated that these cholinium ILs could be used for biomass pretreatment to enhance the enzymatic saccharification of lignocellulosic biomass (Liu et al., 2012; Ninomiya et al., 2013a,b,c, 2014, 2015a,b), as conventional imidazolium IL. Furthermore, our previous study 
demonstrated that cholinium IL was less inhibitory to cellulase enzymes and fermentative microorganisms than imidazolium IL (Ninomiya et al., 2015, 2013a). However, to the best of our knowledge, even for the imidazolium IL, there are few papers reporting the effect of the number of washings on the residual IL amount in the pretreated biomass and the resulting inhibition of enzymatic saccharification of the IL-pretreated biomass, (Li et al., 2009; Li et al., 2013), and there are no papers reporting the above points for the cholinium IL.

Therefore, the present study investigated the effect of the number of washings on the amount of residual cholinium IL in pretreated biomass and the resulting inhibition toward enzymatic saccharification and microbial fermentation of the cholinium IL-pretreated biomass. Moreover, cholinium IL was also examined with regard to biomass pretreatment capability and inhibitory effect on cellulolytic enzymes and fermentation microorganisms. Specifically, choline acetate (ChOAc) was used as the biocompatible cholinium IL. The obtained data for the pretreatment capability, the inhibitory effect on cellulolytic enzymes and fermentation microorganisms, the residual IL amount, the enzymatic saccharification, and the microbial fermentation were compared with data obtained using 1-ethyl-3-methylimidazolium acetate (EmimOAc), the conventional imidazolium IL employed for biomass pretreatment.

\section{Materials and Methods}

\subsection{Biomass, IL, cellulase, and yeast cells}

Bagasse powder (approximately $3 \mathrm{~mm}$ ) was purchased from Toyota Motor Corporation (Miyoshi, Japan) as the lignocellulosic material. The bagasse powder was ground by a mill and then sieved to obtain a powder of $<250 \mu \mathrm{m}$. Microcrystalline 
cellulose (Avicel ${ }^{\circledR}$ PH-101) was purchased from Sigma-Aldrich (St. Louis, MO, USA). Phosphoric acid swollen cellulose (PASC) was prepared from the microcrystalline cellulose according to the method described previously (Wood, 1988). ChOAc was prepared by using a one-pot neutralization method with minor modifications (Yu et al., 2008). EmimOAc was purchased from Kanto Chemical Co., Inc. (Tokyo, Japan).

Commercial cellulase (Cellic $\left.{ }^{\circledR} \mathrm{CTec} 2\right)$, a complex blend of cellulase, hemicellulose, and $\beta$-glucosidase, (batch number VCNI 0008, 106 filter paper units (FPU) per milliliter) was obtained from Novozymes Japan, Ltd (Chiba, Japan). FPU of the cellulase was determined by the method reported by NREL (Adney and Baker, 2008). The yeast strain Saccharomyces cerevisiae MT8-1 (Tajima et al., 1985) was used for ethanol fermentation. All other chemicals were obtained from commercial sources and were of reagent grade.

\subsection{Assay of inhibition by IL on cellulase and yeast activity}

For evaluating the inhibitory effects of IL to cellulase activity, enzymatic saccharification was conducted in a $30 \mathrm{~mL}$ glass vial containing $5 \mathrm{~mL}$ of a mixture of 50 $\mathrm{mM}$ phosphate buffer ( $\mathrm{pH}$ 5.0), IL (EmimOAc or ChOAc) at final concentrations of $0 \%-50 \%(\mathrm{w} / \mathrm{w}), \mathrm{CTec} 2$ at a concentration of $40 \mathrm{FPU} / \mathrm{g}$ of dry biomass, and $0.03 \mathrm{~g}$ of dry weight equivalent of wet PASC as the model substrate. The vial was incubated at $50^{\circ} \mathrm{C}$ for $48 \mathrm{~h}$ using a reciprocal shaker at $130 \mathrm{rpm}$. A sample was taken at $48 \mathrm{~h}$ and then heated at $90^{\circ} \mathrm{C}$ for 5 min to inactivate the enzymes. The heated sample was then centrifuged at $21,500 \times g$ for $1 \mathrm{~min}$ and the supernatant analyzed for glucose by high performance liquid chromatography (HPLC) as described below. The response to the ILs were evaluated based on the relative cellulase activity, which was defined as the 
percentage of the generated glucose at $48 \mathrm{~h}$ in the presence of the IL relative to the generated glucose at $48 \mathrm{~h}$ of a control culture free from the IL. The median effective concentration based on cellulase activity $\left(\mathrm{cEC}_{50}\right)$ was determined as the IL concentration at which the relative cellulase activity was reduced to $50 \%$.

For evaluating the inhibitory effects of IL to yeast, the culture was conducted in a 5-mL test tube containing autoclaved YPD medium $(10 \mathrm{~g} / \mathrm{L}$ of yeast extract, $20 \mathrm{~g} / \mathrm{L}$ of Bacto-peptone, and 20 g/L of glucose; Nacalai Tesque, Kyoto, Japan) supplemented with the IL (EmimOAc or ChOAc) at final concentrations of $0.3 \%-5 \%(\mathrm{w} / \mathrm{w})$ The yeast cells were precultured aerobically at $30^{\circ} \mathrm{C}$ in the test tube containing $5 \mathrm{~mL}$ of YPD medium free from IL. The precultured broth was transferred into a test tube at an initial optical density of 0.1 at a wavelength of $600 \mathrm{~nm}\left(\mathrm{OD}_{600}\right)$. The test tube was incubated at $30^{\circ} \mathrm{C}$ for $48 \mathrm{~h}$ using a reciprocal shaker at $160 \mathrm{rpm}$. The culture broth was sampled at specified culture time points and the $\mathrm{OD}_{600}$ was measured. The response to the IL was evaluated based on the relative growth, which was defined as the percentage of the $\mathrm{OD}_{600}$ at $48 \mathrm{~h}$ in the presence of the IL relative to the $\mathrm{OD}_{600}$ at $48 \mathrm{~h}$ of a control culture free from the IL. The median effective concentration based on yeast growth $\left(\mathrm{yEC}_{50}\right)$ was determined as the IL concentration at which the relative growth was reduced to $50 \%$.

\subsection{IL-assisted pretreatment and washing of biomass}

For biomass pretreatment, $0.5 \mathrm{~g}$ of bagasse powder was added to a $15-\mathrm{mL}$ polypropylene tube (Corning Inc., New York, United States) containing $5 \mathrm{~g}$ of IL (EmimOAc or ChOAc). After vortexing, the biomass/IL mixture in the tube was sonicated for $60 \mathrm{~min}$ at $24 \mathrm{kHz}$ and an emission power of $35 \mathrm{~W}$ using an ultrasonic processor (UP200S with sonotrode S3, Hielscher Ultrasonics GmbH, Teltow, Germany) 
in a water bath maintained at $25^{\circ} \mathrm{C}$.

For washing the IL from the pretreated biomass, $45 \mathrm{~mL}$ of deionized water was then added to the bagasse/IL mixture in a 50-mL tube, which resulted in the precipitation of the biomass. After mixing with inversion for $3 \mathrm{~min}$, the $50-\mathrm{mL}$ tube was centrifuged $(8000 \times g)$ for $10 \mathrm{~min}$ at $25^{\circ} \mathrm{C}$ and the supernatant was removed. The washing procedure was repeated $1-5$ times. For respective washing procedures, the removed supernatant was subjected to measurement of IL concentration using HPLC. The amount of residual IL in the biomass was indirectly determined from the IL amount in the supernatant. The precipitated wet bagasse was stored at $4^{\circ} \mathrm{C}$ until use for subsequent enzymatic saccharification.

\subsection{Saccharification and subsequent fermentation}

Saccharification was performed in a 50-mL polypropylene tube (Corning Inc.) containing the wet pretreated biomass $(0.25 \mathrm{~g}$ as dry weight equivalent $)$ mixed with 5 $\mathrm{mL}$ of $50 \mathrm{mM}$ phosphate buffer $(\mathrm{pH} 5.0)$ and $\mathrm{CTec} 2$ at a concentration of $40 \mathrm{FPU} / \mathrm{g}$ of dry biomass, which resulted in about $3.3 \%$ solid loading. The amount of wet biomass loading was determined from the moisture content of the pretreated biomass with sufficient washing. The tube was set in a heat block (Thermo Block Rotator SN-06BN; Nissinrika Co., Tokyo, Japan) and axially rotated at $35 \mathrm{rpm}$ for $48 \mathrm{~h}$ under a controlled temperature of $50^{\circ} \mathrm{C}$. After enzymatic saccharification for $48 \mathrm{~h}$, the $50-\mathrm{mL}$ tube was centrifuged $(8000 \times g)$ for $10 \mathrm{~min}$ at $4^{\circ} \mathrm{C}$. The liquefied portion $(6 \mathrm{~mL})$ was sampled and used for sugar quantification and subsequent fermentation. For sugar analysis, $1 \mathrm{~mL}$ of the sample was heated at $90^{\circ} \mathrm{C}$ for 5 min to inactivate the enzymes. After centrifugation of the heated sample at $21,500 \times g$ for $1 \mathrm{~min}$, the supernatant was subjected to glucose 
and xylose measurements, as described below. Cellulose saccharification was evaluated as the percentage of cellulose hydrolyzed into glucose compared with cellulose in the original bagasse. In the same manner, hemicellulose saccharification was evaluated as the percentage of hemicellulose hydrolyzed into xylose compared with hemicellulose in the original bagasse.

For fermentation, the yeast was aerobically precultured for 24 hours at $30^{\circ} \mathrm{C}$ and $135 \mathrm{rpm}$ in a 500-mL Sakaguchi flask containing $100 \mathrm{~mL}$ of YPD medium. The yeast cells were collected by centrifugation at $3000 \mathrm{rpm}$ for $5 \mathrm{~min}$ and washed thrice with saline. Fermentation was then performed in a $30-\mathrm{mL}$ glass vial equipped with a gas check valve, which contained a mixture of the $4 \mathrm{~mL}$ of sugar solution obtained in the saccharification step, $1 \mathrm{~mL}$ of quintuple strength YPD medium, and the yeast suspension at a final concentration of $100 \mathrm{~g}$ of wet suspension/L. During the fermentation, samples were collected after 12, 24, and $48 \mathrm{~h}$. After centrifugation of the samples at 21,500 $\times g$ for $1 \mathrm{~min}$, the supernatant was subjected to the ethanol measurement as described below. Ethanol yield on a glucose basis was evaluated as the percentage of ethanol compared with the ethanol theoretically converted from glucose obtained by saccharification. In the same manner, ethanol yield on a bagasse basis was evaluated as the percentage of ethanol compared with the ethanol theoretically converted from cellulose in the original bagasse.

\subsection{Analysis of biomass composition}

The cellulose, hemicellulose, and lignin contents of the original bagasse were determined according to the NREL method (Sluiter et al., 2012) with minor modifications. In brief, $0.1 \mathrm{~g}$ of the sample was mixed with $2 \mathrm{~mL}$ of a $72 \%(\mathrm{v} / \mathrm{v}) \mathrm{H}_{2} \mathrm{SO}_{4}$ 
aqueous solution for $2 \mathrm{~h}$ at RT. The mixture was transferred to a $200-\mathrm{mL}$ Erlenmeyer flask, diluted with $75 \mathrm{~mL}$ of water, and autoclaved at $121^{\circ} \mathrm{C}$ for $15 \mathrm{~min}$. The acid-diluted hydrolysate was filtered, after which the amount of acid-insoluble lignin was gravimetrically determined by measuring the residue on the filter after drying at $100^{\circ} \mathrm{C}$ for $12 \mathrm{~h}$. The amount of acid-soluble lignin was determined from the ultraviolet (UV) absorbance of the filtrate at $205 \mathrm{~nm}$ and an absorption coefficient of $110 \mathrm{~L} \mathrm{~g}^{-1}$ $\mathrm{cm}^{-1}$ (TAPPI, 1991). The sum of the acid-insoluble and acid-soluble lignin was regarded as the total amount of lignin. The amount of sugars in the hydrolysate was determined by HPLC as described below. The amount of cellulose and hemicellulose was calculated from the glucose and xylose content multiplied by the anhydro correction factors of $162 / 180$ and $132 / 150$ respectively.

\subsection{Analysis of glucose, xylose, ethanol, and IL concentrations}

Glucose and xylose concentrations were determined by HPLC equipped with a refractive index (RI) detector (Shimadzu Co., Kyoto, Japan) and a CARBOSep CHO-682 column (Tokyo Chemical Industry Co., Ltd, Tokyo, Japan). The column was run at $85^{\circ} \mathrm{C}$ with a water mobile phase and a flow rate of $0.4 \mathrm{~mL} / \mathrm{min}$. The ethanol concentrations were determined using gas chromatography (GC) equipped with a flame ionization detector (FID) (Shimadzu, Kyoto, Japan) and a CBP-20-S25-050 capillary column (0.32 mm ID, $25 \mathrm{~m}$, Shimadzu, Kyoto, Japan). The injector temperature was $250^{\circ} \mathrm{C}$, and the FID temperature was $280^{\circ} \mathrm{C}$. The column temperature was maintained at $40^{\circ} \mathrm{C}$ for $5 \mathrm{~min}$. Nitrogen was used as the carrier gas at $40 \mathrm{~mL} / \mathrm{min}$. ChOAc and EmimOAc concentrations were determined using HPLC equipped with a refractive index (RI) detector (Shimadzu Co., Kyoto, Japan) and an ICSep-ION-300 column 
(Tokyo Chemical Industry Co., Ltd, Tokyo, Japan). The column was run at $75^{\circ} \mathrm{C}$ with a mobile phase of $4.25 \mathrm{mM} \mathrm{H}_{2} \mathrm{SO}_{4}$ and a flow rate of $0.4 \mathrm{~mL} / \mathrm{min}$.

\section{Results and discussion}

\subsection{Pretreatment capability of ChOAc or EmimOAc for enzymatic saccharification of} bagasse

To examine the pretreatment capability of ChOAc or EmimOAc for subsequent enzymatic hydrolysis of lignocellulosic biomass, bagasse powder was pretreated with each IL and then hydrolyzed by Ctec 2 cellulase for $48 \mathrm{~h}$. Here the pretreated bagasse was washed 5 times to sufficiently remove the IL before saccharification so as to eliminate any inhibitory effect of IL on the cellulolytic enzymes. Especially, in the case of EmimOAc-pretreated bagasse, the gel-like structure of EmimOAc-pretreated bagasse was mashed carefully using a spatula in the washing procedure. Table 1 lists the saccharification percentage of ChOAc- or EmimOAc-pretreated bagasse at $48 \mathrm{~h}$. Without IL pretreatment, the cellulose and hemicellulose saccharification percentages were $24 \%$ and $9 \%$ of the theoretical maximum at $48 \mathrm{~h}$. In contrast, with ChOAc pretreatment, cellulose and hemicellulose saccharification percentages were $90.3 \%$ and 99.9\% at $48 \mathrm{~h}$. These values are approximately the same as those using EmimOAc. These results indicate that ChOAc had a sufficient pretreatment capability for lignocellulosic materials, which was at almost the same level as EmimOAc under the examined pretreatment condition.

EmimOAc has frequently been considered as the most effective IL for cellulose dissolution and pretreatment of enzymatic hydrolysis (Brandt et al., 2013). In general, anions act as hydrogen bond acceptors that interact with the hydroxyl group of cellulose, 
thereby weakening the crystalline structure of cellulose (Remsing et al., 2006). Among the anions of imidazolium ILs, acetate anion has been reported to be the highest hydrogen bond basicity $(\beta)$ and capability of reducing cellulose crystallinity (Brandt et al., 2013). This is why the pretreatment with ChOAc also enhanced the saccharification of cellulose and hemicellulose to the same degree as the pretreatment with EmimOAc

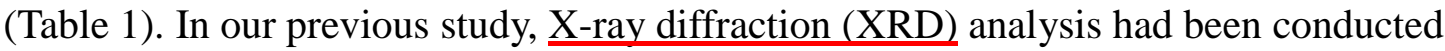
to examine the cellulose crystallinity in the bagasse powder pretreated in ChOAc or EmimOAc with ultrasound irradiation, and a crystallinity index $(\mathrm{CrI})$ was determined on the basis of the XRD spectra. The CrI values were $61.9 \%, 49.1 \%$, and $41.7 \%$ for the original biomass, biomass pretreated in ChOAc, and biomass pretreated in EmimOAc, respectively (Ninomiya et al., 2015b). These results are also generally consistent with the cellulose saccharification results shown in Table 1, suggesting that ChOAc had a substantial pretreatment capability for lignocellulosic biomass. The reason why the saccharification percentage and the CrI values for ChOAc were slightly lower than those for EmimOAc is possibly because the imidazolium cation uniquely interacts with lignin through hydrogen bonding and $\pi-\pi$ interactions (Janesko, 2011), which results in the delignification of biomass via lignin depolymerization (Sun et al., 2014). Moreover, our previous study had also revealed that the composition was almost the same between untreated bagasse powder and bagasse powder pretreated in ChOAc or EmimOAc with ultrasound irradiation under the condition employed in this study (Ninomiya et al., 2015b). Therefore, the pretreatment method used in this study could enhance subsequent saccharification not due to change in biomass composition, but mainly due to change in crystalline structure of cellulose. 


\subsection{Inhibitory effect of ChOAc or EmimOAc on cellulolytic enzymes and yeast cells}

To examine the inhibitory effect of ChOAc or EmimOAc on cellulase enzymes, enzymatic saccharification of the PASC as amorphous cellulose was conducted for $24 \mathrm{~h}$ using Ctec 2 cellulase in the presence of the prescribed concentration of each IL. The $\mathrm{cEC}_{50}$ values were determined from the dose-response relationships between the relative enzyme activity and the IL concentration (Fig. S1A). The $\mathrm{cEC}_{50}$ values were 32 wt\% and $16 \mathrm{wt} \%$ for ChOAc and EmimOAc, respectively (Table 1). To examine the inhibitory effect of ChOAc or EmimOAc on yeast cells, S. cerevisiae MT8-1 was cultured for $48 \mathrm{~h}$ in the presence of the prescribed concentration of each IL. The $\mathrm{yEC}_{50}$ values were determined from the dose-response relationships between relative yeast growth and the IL concentration (Fig. S1B). The $\mathrm{yEC}_{50}$ values were $3.1 \mathrm{wt} \%$ and 0.3 wt\% for ChOAc and EmimOAc, respectively (Table 1). These results indicate that ChOAc shows less inhibitory effect on cellulase and yeast activity compared with EmimOAc under the examined conditions.

Biocatalysts such as enzymes and microbes in IL aqueous solution generally lose their activity due to a higher salt concentration and ionic strength. Thus, a more biocompatible IL was needed to perform the saccharification of IL-pretreated biomass with fewer post-pretreatment washings. The inhibitory effects of ChOAc on cellulase and yeast cells were significantly lower than those of EmimOAc (Table 1) because the cholinium cation was reported to be more intrinsically biocompatible than the imidazolium cation (Ninomiya, 2015, 2013b; Petkovic et al., 2010; Hou et al., 2013).

\subsection{Residual amount of IL in ChOAc or EmimOAc-pretreated biomass with a different number of washings}


The morphology of biomass was observed after the IL-pretreatment followed by washing the prescribed number of times. Figure S2B-E shows the bagasse powder, which was pretreated with EmimOAc or ChOAc and then washed once or 5 times. In the case of EmimOAc, pretreated bagasse exhibited a gel-like morphology irrespective of the number of wash times, 1 or 5 (Fig. S2B and D). On the other hand, in the case of ChOAc, pretreated bagasse exhibited a powdered morphology (Fig. S2C and E), like non-treated bagasse (Fig. S2A). It was considered that these different morphologies for EmimOAc and ChOAc-pretreated bagasse would lead to differences in washing efficiency and differences in the residual amount of IL in the IL-pretreated biomass.

To examine the amount of residual IL in the ChOAc or EmimOAc-pretreated biomass, the bagasse was pretreated with each IL and then washed 1-5 times. Figure 1 shows the residual IL percentage in pretreated biomass with a different number of post-pretreatment wash times. Irrespective of the ILs, the percentages decreased as the number of washing times increased, reaching the approximate plateaus at 5 washings. The residual IL percentage in the case of ChOAc was lower than the cases for EmimOAc throughout the number of wash times. In the case of EmimOAc, the residual IL percentages were $46 \%$ and $9 \%$ at 1 and 5 wash times, respectively. On the other hand, in the case of ChOAc, the residual IL percentages were $20 \%$ and $4 \%$ at 1 and 5 wash times, respectively. These results indicate that ChOAc was more easily washed away from the pretreated bagasse compared than EmimOAc.

The biomass-derived gel is actually generated when antisolvent (water or alcohol) is added into the biomass/IL solution to re-precipitate the biomass once it is solubilized into IL (Dibble et al., 2011). The biomass-derived gel is so stiff that it cannot be completely dispersed in the antisolvent and the IL cannot be easily separated from the 
biomass-gel, which causes a loss of the expensive IL and an inhibition toward cellulolytic enzymes and fermentation microorganisms in the subsequent processes. In the present study, it was found that ChOAc generated less biomass-gel and less IL remained in the pretreated bagasse compared with that for EmimOAc-treated biomass (Fig. 1). This may be because EmimOAc has a relatively higher capability to reduce the cellulose crystallinity and to swell the biomass than ChOAc. The residual IL percentage was $9 \%$ at 5 wash times in the case of EmimOAc, whereas the residual IL percentage was reduced to the same level with only 2 wash times in the case of ChOAc (Fig. 1). This means that pretreatment with ChOAc can reduce the amount of wastewater.

\subsection{Saccharification and subsequent fermentation of ChOAc or EmimOAc-pretreated} biomass with a different number of wash times

To examine the saccharification efficiency of ChOAc or EmimOAc-pretreated biomass with a different the number of post-pretreatment wash times, the pretreated bagasse powders were washed 1-5 times, and then subjected to enzymatic saccharification using Ctec 2 for $48 \mathrm{~h}$. Figure $2 \mathrm{~A}$ and B show the cellulose and hemicellulose saccharification percentages obtained at $48 \mathrm{~h}$ of saccharification of ChOAc or EmimOAc-pretreated bagasse with a different number of wash times. The saccharification percentages increased as the number of washing times increased, irrespective of the ILs. The saccharification percentages for the case of ChOAc were higher than those for the cases of EmimOAc throughout the 5 wash times. In the case of EmimOAc, the cellulose and hemicellulose percentages were $73 \%$ and $33 \%$ at 5 wash times, respectively. Whereas in the case of ChOAc, cellulose, and hemicellulose, the saccharification percentage was $88 \%$ and $66 \%$ at 5 wash times, respectively. Even at 2 
wash times, the cellulose and hemicellulose saccharification percentages for ChOAc reached $75 \%$ and $43 \%$, respectively, although the cellulose and hemicellulose saccharification percentages were just $44 \%$ and $5 \%$, respectively, in the case of EmimOAc.

To examine the fermentation efficiency of sugars saccharified from ChOAc or EmimOAc-pretreated biomass with a different number of post-pretreatment wash times, the pretreated bagasse powder, which was washed 1-5 times, was enzymatically hydrolyzed for $48 \mathrm{~h}$, followed by ethanol fermentation using yeast MT8-1 for another $48 \mathrm{~h}$. Figure $2 \mathrm{C}$ shows ethanol yield on a glucose basis obtained at $48 \mathrm{~h}$ of fermentation from glucose generated by saccharification of ChOAc or EmimOAc-pretreated bagasse with a different number of wash times. The ethanol yield enhanced along with the number of washing times, reaching saturated values, irrespective of the ILs. The ethanol yield for the case of ChOAc was higher than those for the cases of EmimOAc at 1 and 2 wash times. The ethanol yields at 2 wash times were $73 \%$ and $54 \%$ in the cases of ChOAc and EmimOAc, respectively. The ethanol yields were almost at the constant level of around $75 \%$ irrespective of the ILs at 3-5 wash times.

Figure 3 shows the overall ethanol yield on the original bagasse basis obtained at $48 \mathrm{~h}$ of fermentation from glucose generated by saccharification of ChOAc or EmimOAc-pretreated bagasse with a different number of wash times. As can be expected from the results in Figure 3, the overall ethanol yield enhanced along with the number of washing times, reaching the saturated values at approximately $60 \%$, irrespective of the ILs. The ethanol yield for the case of ChOAc was higher than those for the cases of EmimOAc at 1-3 wash times. The overall ethanol yields at 2 wash times were $60 \%$ and $24 \%$ for the case of ChOAc and EmimOAc, respectively. Four 
post-pretreatment washing times were required to reach the saturated value in the case of EmimOA. These results indicate that ChOAc-pretreated bagasse could be saccharified and fermented with fewer wash times than EmimOAc-pretreated bagasse.

The present study, for the first time, compared cholinium IL (ChOAc) and imidazolium IL (EmimOAc) systematically from the viewpoint of the pretreatment capability of biomass (Table 1), the inhibitory effects on cellulase and yeast (Table 1), the residual percentage in pretreated biomass after post-pretreatment washing (Fig. 1), and the saccharification and subsequent fermentation (Figs. 2 and 3). It was revealed that ChOAc-pretreated bagasse could be saccharified and fermented with fewer wash times than EmimOAc-pretreated bagasse (Figs. 2 and 3). This was because the ChOAc showed almost the same capability of biomass pretreatment for saccharification (Table 1), the less inhibitory effects on cellulase and yeast (Table 1), and the less residual amounts in the pretreated bagasse due to less biomass-derived gel (Fig. 1). We therefore suggest that ChOAc is a more favorable IL for biomass pretreatment prior to saccharification and fermentation since it can reduce the number of post-pretreatment wash times and reduce the resultant wastewater of diluted IL aqueous solution under the examined conditions.

Figure 4 shows the dose-response relationships for cellulose saccharification percentage, hemicellulose saccharification percentage and ethanol yield on a glucose basis, which were plotted against the residual IL concentration in the enzymatic reaction mixture and yeast culture media. This Fig. 4 was obtained by the combined use of the data shown in Figs. 2 and 1E. It confirmed that ChOAc shows less inhibitory effect on cellulose and hemicellulose saccharification with Ctec2 cellulase than EmimOAc (Fig. 4A and B). These dose-response profiles for Ctec 2 cellulase activity were more 
sensitive than those shown in Figure S1A, since the substrate was not PASC but IL-treated bagasse. On the other hand, the dose-response profiles for ethanol yield on a glucose basis overlapped between the cases of ChOAc and EmimOAc (Fig. 4C), although ChOAc showed less inhibitory effect on yeast growth activity than EmimOAc (Fig. S1B). This is because the inoculum size was larger in the case of ethanol fermentation (initial $\mathrm{OD}_{600}=10$ ) compared with the case of the growth inhibition assay (initial $\mathrm{OD}_{600}=0.1$ ). Above all, we suggest that $\mathrm{ChOAc}$ is a promising alternative to EmimOAc for the saccharification and fermentation of IL-pretreated biomass with fewer post-pretreatment washing and less wastewater, although the positive effect of ChOAc was mainly seen in saccharification under the examined conditions in the present study.

Based on the techno-economic analysis for the bioethanol production process based on IL-pretreatment, enzymatic saccharification, and yeast fermentation (Klein-marcuschamer et al., 2011), it is necessary to attain the high IL recovery ratio (over 97\%) and high biomass loading to IL (33-50 wt\%) during the pretreatment step as well as the low IL cost (less than $\$ 2.5 / \mathrm{kg}$ ) to ensure the minimum ethanol selling price (MESP) at \$5/gal. As for the high IL recovery ratio, our previous study (Ninomiya et al., 2015a) demonstrated that the recovery ratio of the ionic liquid (ChOAc) was about $95 \%$ and its pretreatment capability was kept during 5 cycles of recycle. As for the high biomass loading to IL during the pretreatment step, our previous study (Ninomiya et al., 2013b) demonstrated that ChOAc pretreatment at a biomass loading up to $25 \mathrm{wt} \%$ did not deteriorate pretreatment efficiency (i.e., cellulose saccharification), but the pretreatment efficiency decreased to less than half when biomass loading was increased to $50 \mathrm{wt} \%$. Therefore, it is important to attain the sufficient pretreatment efficiency by a 
combined use of ChOAc and microwave irradiation and so on, even when the biomass loading is $50 \mathrm{wt} \%$. Reducing both the ChOAc amount for biomass pretreatment and the number of washing times after pretreatment will make the IL-assisted bioethanol production process more economically and environmentally friendly because of less IL use and less wastewater discharge.

\section{Conclusions}

The present study compared ChOAc and EmimOAc from the viewpoint of the pretreatment capability of biomass, the inhibitory effects on cellulase and yeast, the residual percentage in pretreated bagasse after post-pretreatment washing, and the saccharification and subsequent fermentation of pretreated bagasse. The ChOAc showed almost the same capability of pretreatment for saccharification, less residual IL in the pretreated bagasse, and less inhibitory effects on cellulase and yeast. As a result, ChOAc-pretreated bagasse could be saccharified and fermented even with fewer wash times than EmimOAc-pretreated bagasse. This could reduce the wastewater from the diluted IL aqueous solution.

\section{Acknowledgments}

This study was supported in part by an Advanced Low Carbon Technology Research and Development Program (ALCA) (Grant number 2100040) from Japan 
Science and Technology Agency. This study was supported in part by a Center of Innovation Science and Technology based Radical Innovation and Entrepreneurship Program (COIstream) from the Japan Science and Technology Agency. This study was supported in part by a Cross-ministerial Strategic Innovation Promotion Program (SIP) from Japan Science and Technology Agency.

\section{References}

1. Adney, B., Baker, J., 2008. Measurement of cellulase activities, Technical Report, NREL/TP-510-42628, National Renewable Energy Laboratory, Golden, CO.

2. Brandt, A., Gräsvik, J., Hallett, J.P., Welton, T., 2013. Deconstruction of lignocellulosic biomass with ionic liquids. Green Chem. 15, 550-583.

3. Dadi, A.P., Varanasi, S., Schall, C.A., 2006. Enhancement of cellulose saccharification kinetics using an ionic liquid pretreatment step. Biotechnol. Bioeng. 95, 904-910.

4. Datta, S., Holmes, B., Park, J.I., Chen, Z., Dibble, D.C., Hadi, M., Blanch, H.W., Simmons, B.A., Sapra, R., 2010. Ionic liquid tolerant hyperthermophilic cellulases for biomass pretreatment and hydrolysis. Green Chem. 12, 338-345.

5. Dibble, D.C., Li, C., Sun, L., George, A., Cheng, A., Çetinkol, Ö.P., Benke, P., Holmes, B.M., Singh, S., Simmons, B.A., 2011. A facile method for the recovery of ionic liquid and lignin from biomass pretreatment. Green Chem. 13, 3255-3264.

6. Fukaya, Y., lizuka, Y., Sekikawa, K., Ohno, H., 2007. Bio ionic liquids: room temperature ionic liquids composed wholly of biomaterials. Green Chem. 9, $1155-1157$.

7. Hou, X.D., Liu, Q.P., Smith, T.J., Li, N., Zong, M.H., 2013. Evaluation of toxicity 
and biodegradability of cholinium amino acids ionic liquids. PLoS One. 8, e59145.

8. Hu, S., Jiang, T., Zhang, Z., Zhu, A., Han, B., Song, J., Xie, Y., Li, W., 2007.

Functional ionic liquid from biorenewable materials: synthesis and application as a catalyst in direct aldol reactions. Tetrahedron Lett. 48, 5613-5617.

9. Janesko, B.G., 2011. Modeling interactions between lignocellulose and ionic liquids using DFT-D. Phys. Chem. Chem. Phys. 13, 11393-11401.

10. Klein-marcuschamer, D., Simmons, B.A., Blanch, H.W., 2011. Techno-economic analysis of a lignocellulosic ethanol biorefinery with ionic liquid pretreatment, Biofuels Bioproducs and Biorefining. 5, 562-569.

11. Li, C., Knierim, B., Manisseri, C., Arora, R., Scheller, H.V., Auer, M., Vogel, K.P., Simmons, B.A., Singh, S., 2010. Comparison of dilute acid and ionic liquid pretreatment of switchgrass: Biomass recalcitrance, delignification and enzymatic saccharification. Bioresour. Technol. 101, 4900-4906.

12. Li, C., Cheng, G., Balan, V., Kent, M.S., Ong, M., Chundawat, S.P.S., Sousa, L.C., Melnichenko, Y.B., Dale, B.E., Simmons, B.A., Singh, S., 2011. Influence of physico-chemical changes on enzymatic digestibility of ionic liquid and AFEX pretreated corn stover. Bioresour. Technol. 102, 6928-6936.

13. Li, C., Tanjore, D., He, W., Wong, J., Gardner, J.L., Sale, K.L., Simmons, B.A., Singh, S., 2013. Scale-up and evaluation of high solid ionic liquid pretreatment and enzymatic hydrolysis of switchgrass. Biotechnol. Biofuels. 6, 154.

14. Li, Q., He, Y.-C., Xian, M., Jun, G., Xu, X., Yang, J.-M., Li, L.Z., 2009. Improving enzymatic hydrolysis of wheat straw using ionic liquid 1-ethyl-3-methyl imidazolium diethyl phosphate pretreatment. Bioresour. Technol. 100, 3570-3575.

15. Liu, Q.P., Hou, X.D., Li, N., Zong, M.H., 2012. Ionic liquids from renewable 
biomaterials: synthesis, characterization and application in the pretreatment of biomass. Green Chem. 14, 304-307.

16. Ninomiya, K., Yamauchi, T., Kobayashi, M., Ogino, C., Shimizu, N., Takahashi, K., 2013a. Cholinium carboxylate ionic liquids for pretreatment of lignocellulosic materials to enhance subsequent enzymatic saccharification. Biochem. Eng. J. 71, $25-29$.

17. Ninomiya, K., Soda, H., Ogino, C., Takahashi, K., Shimizu, N., 2013b. Effect of ionic liquid weight ratio on pretreatment of bamboo powder prior to enzymatic saccharification. Bioresour. Technol. 128, 188-192.

18. Ninomiya, K., Ohta, A., Omote, S., Ogino, C., Takahashi, K., Shimizu. N., 2013c. Combined use of completely bio-derived cholinium ionic liquids and ultrasound irradiation for the pretreatment of lignocellulosic material to enhance enzymatic saccharification, Chem. Eng. J. 215-216, 811-818.

19. Ninomiya, K., Yamauchi, T., Ogino, C., Shimizu, N., Takahashi, K., 2014. Microwave pretreatment of lignocellulosic material in cholinium ionic liquid for efficient enzymatic saccharification. Biochem Eng. J. 90, 90-95.

20. Ninomiya, K., Inoue, K., Aomori, Y., Ohnishi, A., Ogino, C., Shimizu, N., Takahashi, K., 2015a. Characterization of fractionated biomass component and recovered ionic liquid during cholinium ionic liquid-assisted pretreatment process with its recycle. Chem Eng. J. 259, 323-329.

21. Ninomiya, K., Kohori, A., Tatsumi, M., Osawa, K., Endo, T., Kakuchi, R., Ogino, C., Shimizu, N., Takahashi, K., 2015b. Ionic liquid/ultrasound pretreatment and in situ enzymatic saccharification of bagasse using biocompatible cholinium ionic liquid, Bioresour. Technol. 176, 169-174. 
22. Ouellet, M., Datta, S., Dibble, D.C., Tamrakar, P.R., Benke, P.I., Li, C., Singh, S., Sale, K.L., Adams, P.D., Keasling, J.D., Simmons, B.A., Holmes, B.M., Mukhopadhyay, A., 2011. Impact of ionic liquid pretreated plant biomass on Saccharomyces cerevisiae growth and biofuel production. Green Chem. 13, $2743-2749$.

23. Petkovic, M., Ferguson, J.L., Gunaratne, H.N., Ferreira, R., Leitao, M.C., Seddon, K.R., Rebelo, L.P.N., Silva Pereira, C., 2010. Novel biocompatible cholinium-based ionic liquids - Toxicity and biodegradability. Green Chem. 12, 643-649.

24. Remsing, R.C., Swatloski, R.P., Rogers, R.D., Moyna, G., 2006. Mechanism of cellulose dissolution in the ionic liquid 1-n-butyl-3-methylimidazolium chloride: a ${ }^{13} \mathrm{C}$ and ${ }^{35 / 37} \mathrm{Cl}$ NMR relaxation study on model systems. Chem. Commun. $1271-1273$.

25. Sluiter, A., Hames, B., Ruiz, R., Scarlata, C., Sluiter, J., Templeton, D., Crocker, D., 2012. Determination of structural carbohydrates and lignin in biomass determination of structural carbohydrates and lignin in biomass, Technical Report, NREL/TP-510-42618, National Renewable Energy Laboratory, Golden, CO.

26. Sun, N., Parthasarathi, R., Socha, A.M., Shi, J., Zhang, S., Stavila, V., Sale, K.L., Simmons, B.A., Singh, S., 2014. Understanding pretreatment efficacy of four cholinium and imidazolium ionic liquids by chemistry and computation. Green Chem. 16, 2546-2557.

27. Swatloski, R.P., Spear, S.K., Holbrey, J.D., Rogers, R.D., 2002. Dissolution of cellose with ionic liquids. J. Am. Chem. Soc. 124, 4974-4975.

28. Tajima, M., Nogi, Y., Fukusawa, T., 1985. Primary structure of the Saccharomyces cerevisiae GAL7 gene, Yeast. 1, 67-77. 
29. TAPPI UM 250, 1991. Acid-soluble lignin in wood and pulp, in: TAPPI Useful

Methods. Tappi, Atlanta.

30. Wood, T.M., 1988. Preparation of crystalline, amorphous, and dyed cellulase substrates, Methods Enzymol. 160, 19-25.

31. Yu, Y., Lu, X., Zhou, Q., Dong, K., Yao, H., Zhang, S., 2008. Biodegradable naphthenic acid ionic liquids: synthesis, characterization, and quantitative structure-biodegradation relationship. Chem. Eur. J. 14, 11174-11782. 


\section{Figure captions}

Fig. 1 Percentages of residual IL in IL-pretreated bagasse with different number of wash times. Open bars: EmimOAc, and closed bars: ChOAc. The error bars indicate the standard error from three independent experiments.

Fig. 2 (A) Cellulose saccharification percentage and (B) hemicellulose saccharification percentage obtained using data from the 48-h saccharification of IL-pretreated bagasse with different number of wash times. The $100 \%$ cellulose and hemicellulose saccharification corresponds to $13.8 \mathrm{~g} / \mathrm{L}$ glucose and $8.6 \mathrm{~g} / \mathrm{L}$ xylose, respectively. (C) Ethanol yield on a saccharified glucose basis. The yield was obtained using data from the 48-h fermentation of glucose saccharified from IL-pretreated bagasse with different number of wash times. Open bars: EmimOAc, and closed bars: ChOAc. The error bars indicate the standard error from three independent experiments.

Fig. 3 Ethanol yield on an original bagasse basis. The yield was obtained using data from the 48-h fermentation of glucose saccharified from IL-pretreated bagasse with different number of wash times. The $100 \%$ ethanol yield corresponds to $5.6 \mathrm{~g} / \mathrm{L}$ ethanol. Open bars: EmimOAc, and closed bars: ChOAc. The error bars indicate the standard error from three independent experiments.

Fig. 4 Correlation plots of (A) cellulose saccharification, (B) hemicellulose saccharification, and (C) ethanol yield on a saccharified glucose basis against 
residual IL concentration, which were obtained for different number of wash times. Open keys: EmimOAc, and closed keys: ChOAc. The error bars indicate the standard error from three independent experiments. 
Table 1. Pretreatment capability and inhibitory effect of EmimOAc or ChOAc.

\begin{tabular}{|c|c|c|c|c|}
\hline \multirow{2}{*}{ IL } & \multicolumn{2}{|c|}{$\begin{array}{l}\text { Pretreatment capability of IL } \\
\text { on bagasse powder }^{\text {a }}\end{array}$} & \multicolumn{2}{|c|}{$\begin{array}{l}\text { Inhibitory effect of IL } \\
\text { on cellulase and yeast }{ }^{b}\end{array}$} \\
\hline & $\begin{array}{l}\text { Cellulose } \\
\text { saccharification } \\
\text { percentage [\%] }\end{array}$ & $\begin{array}{l}\text { Hemicellulose } \\
\text { saccharification } \\
\text { percentage [\%] }\end{array}$ & $\begin{array}{l}\mathrm{cEC}_{50} \\
{[\mathrm{wt} \%]}\end{array}$ & $\begin{array}{l}\mathrm{yEC}_{50} \\
{[\mathrm{wt} \%]}\end{array}$ \\
\hline EmimOAc & $102.3 \pm 0.3$ & $104.7 \pm 4.0$ & $16.2 \pm 0.3$ & $0.3 \pm 0.01$ \\
\hline ChOAc & $90.3 \pm 5.0$ & $99.9 \pm 6.2$ & $32.3 \pm 1.3$ & $3.1 \pm 0.11$ \\
\hline
\end{tabular}

The data were expressed as mean \pm standard error from three independent experiments. $a$ Pretreatment of bagasse powder was carried out by sonication in each IL at $25^{\circ} \mathrm{C}$ for $60 \mathrm{~min}$. The cellulose and hemicellulose saccharification percentage was obtained at $48 \mathrm{~h}$ of enzymatic reaction of pretreated bagasse with sufficient post-pretreatment wash.

$b$ The $\mathrm{cEC}_{50}$ value was determined based on cellulase activity of Ctec 2 using PASC as substrate. The $\mathrm{yEC}_{50}$ value was determined based on growth activity of yeast $S$. cerevisiae MT8-1. 


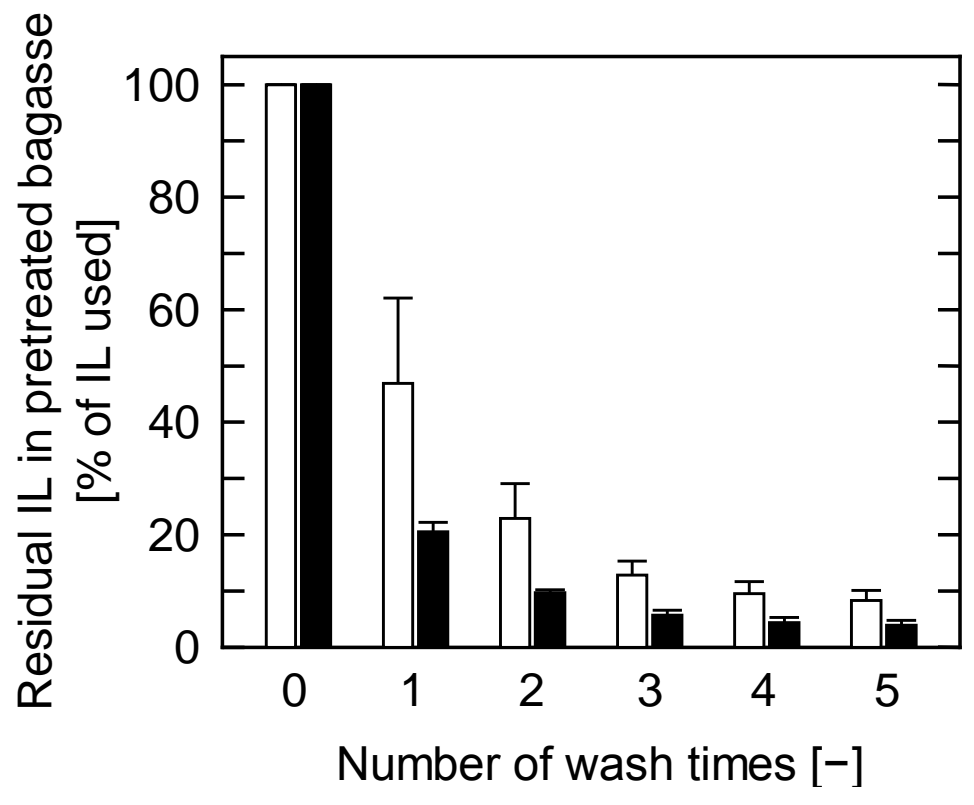

Fig. 1 

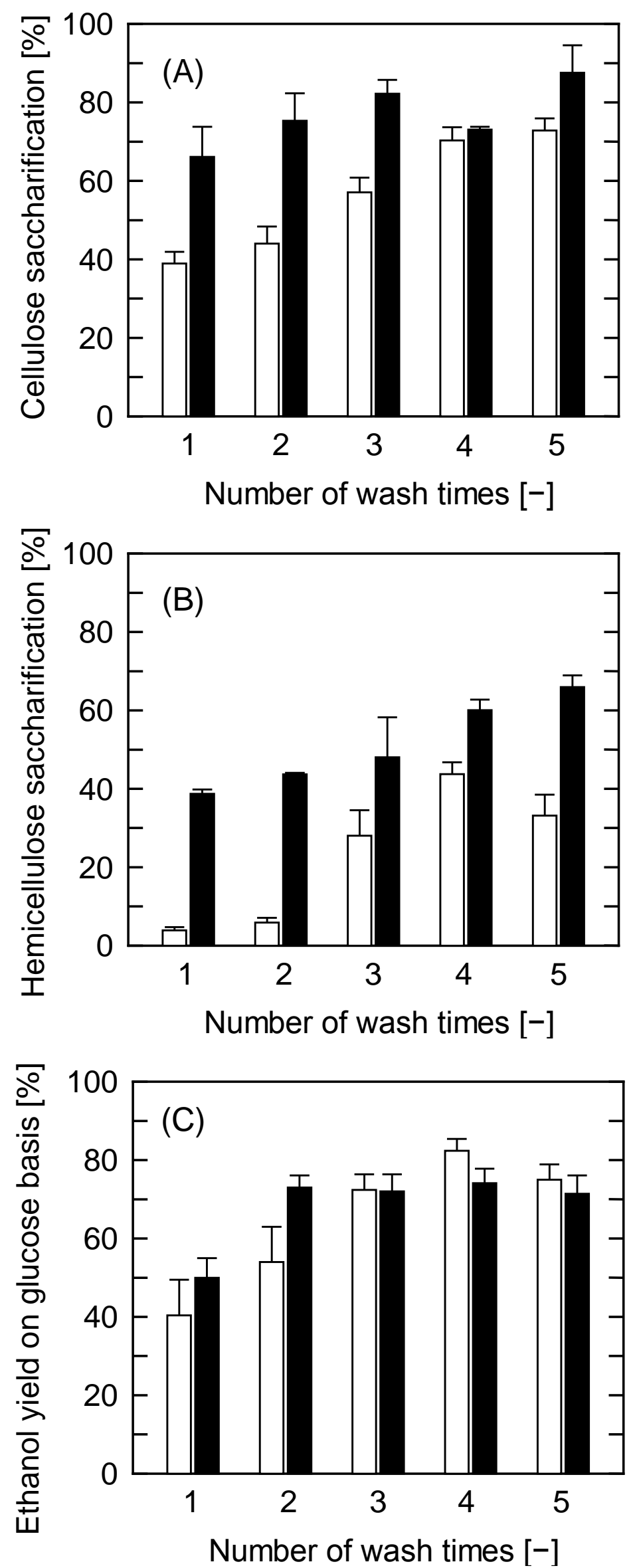

Fig. 2 


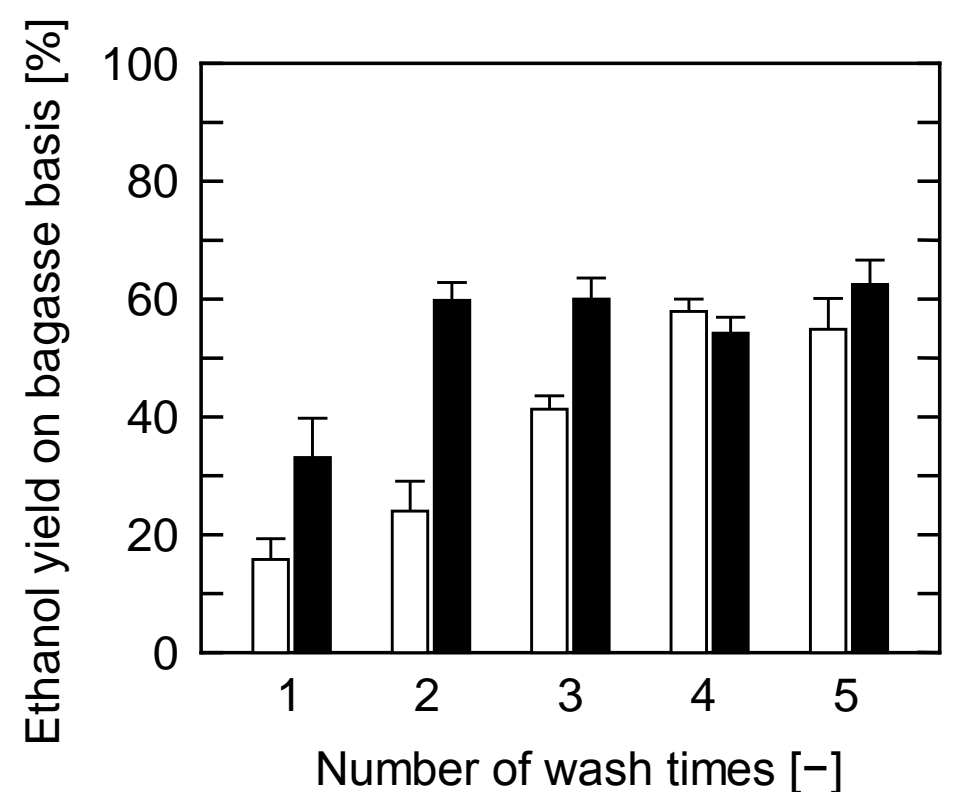

Fig. 3 

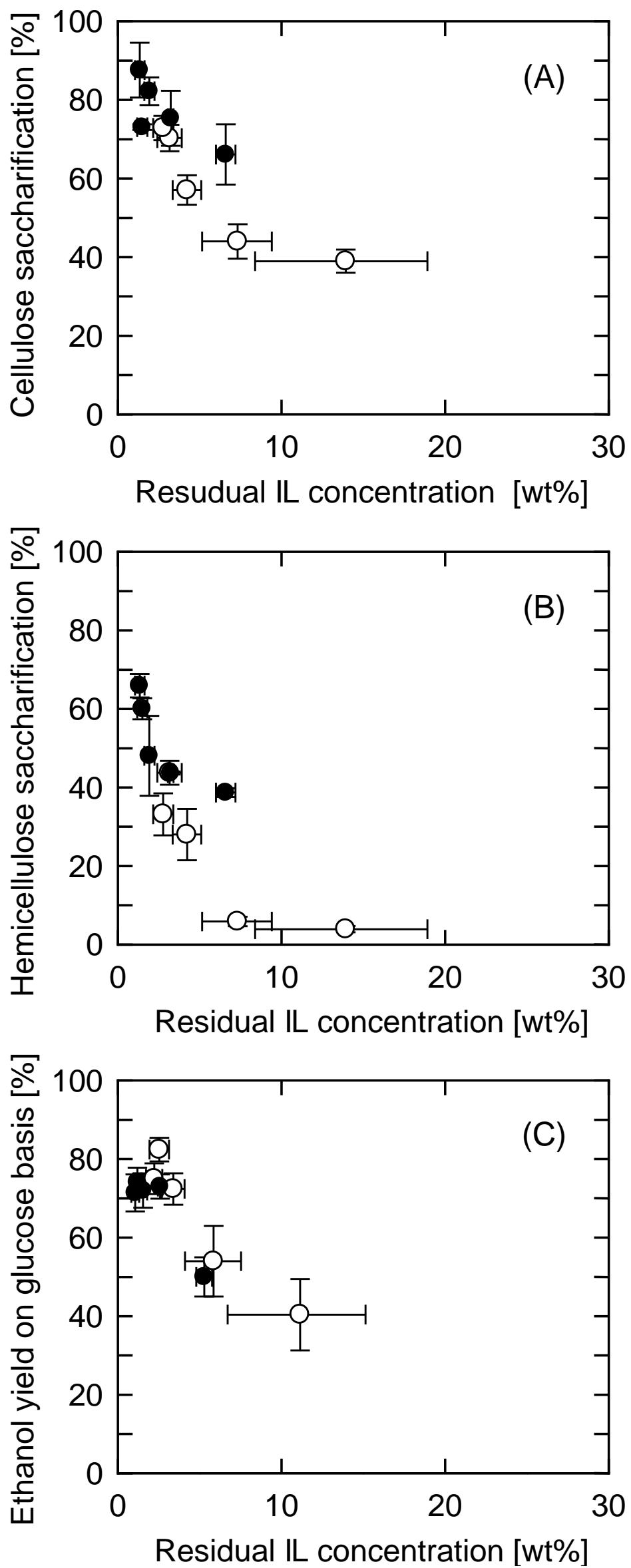

Fig. 4 
Graphical Abstract (for review)

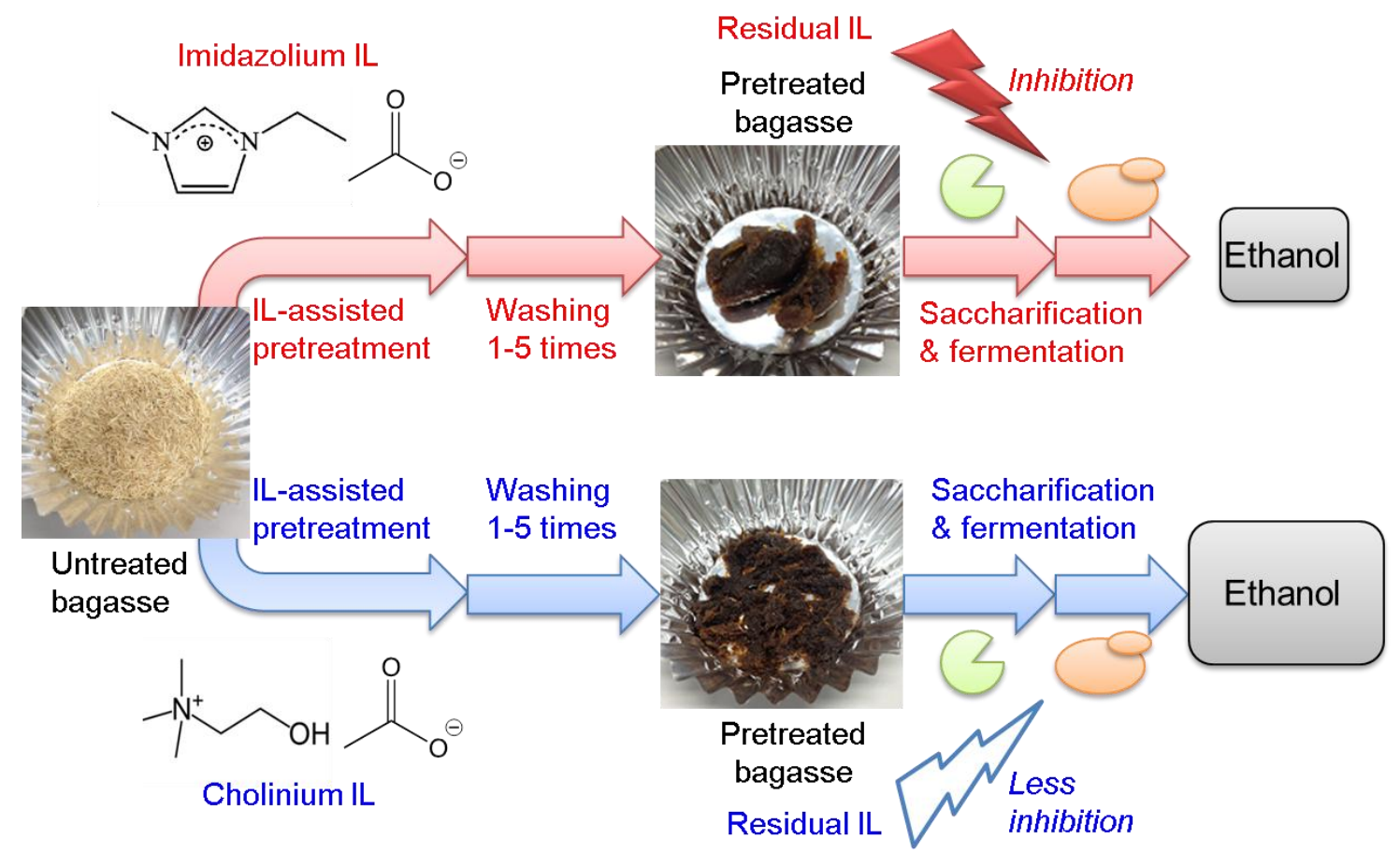

